\title{
Spin-glass model with partially annealed asymmetric bonds.
}

\author{
A.E. Allahverdyan, D.B. Saakian \\ Yerevan Physics Institute \\ Alikhanian Brothers St.2, Yerevan 375036, Armenia
}

\begin{abstract}
We have considered the two-spin interaction spherical spin-glass model with asymmetric bonds (coupling constants). Besides the usual interactions between spins and bonds and between the spins and a thermostat with temperature $T_{\sigma}$ there is also an additional factor: the bonds are not assumed random a priori but interact with some other thermostat at the temperature $T_{J}$. We show that when the bonds are frozen with respect to the spins a first order phase transition to a spin-glass phase occurs, and the temperature of this transition tends to zero if $T_{J}$ is large. Our analytical results show that a spin-glass phase can exist in mean-field models with nonrelaxational dynamics.
\end{abstract}


Investigation of nonequilibrium stationary states for systems without detailed balance is an important problem. Systems of this type are widely used in nonequilibrium thermodynamics [1, 2], biology and biophysics [3], theory of artificial neural networks [4], etc. [5.

If detailed balance (or potential conditions) holds, then the stationary state of a macroscopic system can be described by the Gibbs distribution (maybe with some generalizations), which is independent from details of the dynamics [6, 7]. In the opposite case such a universal and simple distribution does not exist, and the situation is strongly dependent on the details of dynamics and the concrete form of detailed balance violation. In general, we have in a stationary state without detailed balance only timetranslation invariance [6, 7]: average multitime quantities $C\left(t_{1}, . ., t_{n}\right)$ (time ordering is assumed $t_{1} \geq \ldots \geq t_{n}$ ) depend only on the corresponding time differences $t_{k}-t_{l}, k<l$ (for example, $C(t)=$ const, $C\left(t_{1}, t_{2}\right)=C\left(t_{1}-t_{2}\right), \ldots$ )

In the present paper we consider the case when the detailed balance condition is violated by nonconservative forces of some specific type: the mean-field spherical spinglass model with asymmetric bonds (coupling constants). This type of nonconservative forces was inspired by applications in the theory of artificial neural networks [4], but it has also some independent meaning as the model for a open spin-glass system. The model was introduced in [9], where was shown that random frozen uncorrelated asymmetric gaussian bonds (even when the asymmetry is small but generic) totally break finitetemperature spin-glass transition. The second important step in this direction was done in [10]. Here multispin interaction spherical spin-glass was considered, and by numerical methods was shown that indeed the Langevin dynamics, which is started from the typical initial conditions, tends to the paramagnetic state for any nonzero temperature. But if the initial conditions are chosen in some special way, then finite-temperature spin-glass phase transition to a metastable state is possible. Gugliandolo et. al. [10] proposed that this difference between two-spin and multispin interaction models arises due to the different structure of the phase spaces when detailed balance holds (i.e., the bonds are symmetric). Indeed in the two-spin interaction model the spin-glass phase is marginally 
stable, hence there is a possibility to destroy the phase transition. In contrast, the multispin interaction model has low-lying totally stable states [11, 12, 14].

In our model the coupling constants interact with the spins and with a thermal bath at the temperature $T_{J}$. Generally speaking, can be $T_{J} \neq T_{\sigma}(\equiv T)$. There are several reasons for introducing interactions of this type. A typical example of a two-temperature system is a nonequilibrium electron-proton plasma. Due to the large difference between electron and proton masses energy transfer between the two components can be neglected in some range of times. Protons and electrons then go to equilibrium independently and can have different temperatures. In general, a nonequilibrium many-component systems with slow energy transfer between the components can, in some range of times, be described by introducing several temperatures. Another reason is that: It is well known that in some cases and in some sense neural networks can be described by spin-glass models. The spins and coupling constants are identified as neurons and synaptic connections. But from a physiological point of view the nonlinear interactions with the neurons, and varying the synaptic connections with external environment is a very important part of recognition and memory.

At first, spin-glass models with slow varying bonds were considered by Horner [8] t. Here the model with the detailed balance condition is considered, and the slow motion of bonds is used as some method for obtaining correct long-time limits from dynamical equations. Other methods for this purpose are also developed by Horner and co-workers 12 .

The Langevin equations for the model have the following form:

$$
\begin{gathered}
\tau \partial_{t} \sigma_{i}=-r \sigma_{i}-\gamma \beta \sum_{j} J_{i j} \sigma_{j}+\eta_{i}(t), \quad\left\langle\eta_{i}(t) \eta_{j}\left(t^{\prime}\right)\right\rangle=2 \tau \delta_{i j} \delta\left(t-t^{\prime}\right), \\
\tau_{J} \partial_{t} J_{i j}=\gamma \beta_{J} \sigma_{i} \sigma_{j}-2 v \beta_{J} J_{i j}+\eta_{i j}(t), \quad\left\langle\eta_{i j}(t) \eta_{i^{\prime} j^{\prime}}\left(t^{\prime}\right)\right\rangle=2 \tau_{J} \delta_{i i^{\prime}} \delta_{j j^{\prime}} \delta\left(t-t^{\prime}\right),
\end{gathered}
$$

where the bonds (spins) interact with the thermal bath at temperature $T_{J}\left(T_{\sigma}\right), \gamma=$ $\underline{\sqrt{2 / N}}$ is the standard norm-factor for mean-field models, $r$ is the lagrangian factor for

\footnotetext{
${ }^{1}$ We thank referee for pointing these references.
} 
the spherical constraint $\sum_{i} \sigma_{i}^{2}=N$ (here $N$ is the number of spins), $J_{i j} \neq J_{j i}$, and $v J_{i j}^{2}$ is the potential energy for a coupling constant.

If the bonds are symmetric a priori, then Eqs. (11) and (2) can be viewed as equations generated by the mean-field Hamiltonian

$$
H=\gamma \sum_{i j} J_{i j} \sigma_{i} \sigma_{j}+v \sum_{i j} J_{i j}^{2}
$$

It is the Langevin dynamics of the well-known two-spin spherical model where the (symmetric) bonds interact with the thermal bath at the temperature $T_{J}$ [13]. In model considered in this paper asymmetric and symmetric bonds have the similar potential energy, and are subjected to the different thermal histories of the same thermostat.

The analysis of Eqs. (11) and (2) simplifies considerably in the thermodynamic limit when $N \rightarrow \infty$, where the dynamics of the system can be described by a self-consistent equation involving a single spin only. This is achieved by introducing generating functional for the Langevin dynamics [16]. Because our interest is in the dynamics of the spins only, the integration by the bonds can be taken in the generating functional.

The resulting mean-field equations read

$$
\begin{aligned}
& \left(\tau \partial_{t}+r\right) \sigma(t)=\tilde{\mu} \int_{-\infty}^{t} d \bar{t} e^{-\alpha(t-\bar{t})} C(\bar{t}, t) \sigma(\bar{t})+\eta(t), \\
& \left\langle\eta(t) \eta\left(t^{\prime}\right)\right\rangle=2 \tau \delta\left(t-t^{\prime}\right)+\mu \exp \left(-\alpha\left|t-t^{\prime}\right|\right) C\left(t, t^{\prime}\right),
\end{aligned}
$$

where $\alpha=2 v \beta_{J} / \tau_{J}, \tilde{\mu}=2 \beta \beta_{J} / \tau_{J}, \mu=\beta^{2} / v \beta_{J}$. Our purpose here is to get equations for the correlation function $C\left(t, t^{\prime}\right)=\left\langle\sigma(t) \sigma\left(t^{\prime}\right)\right\rangle$ and the response function $G\left(t, t^{\prime}\right)=$ $\left\langle\partial \sigma(t) / \partial \beta h\left(t^{\prime}\right)\right\rangle$. Because the initial time $\rightarrow-\infty$ (it is one of the limits of integration in Eq. (3)) the equilibrium regime is expected

$$
C\left(t, t^{\prime}\right)=C\left(t-t^{\prime}\right), \quad G\left(t, t^{\prime}\right)=G\left(t-t^{\prime}\right)
$$

It should be stressed again that the initial time tends to minus infinity after the thermodynamic limit. The resulting equations are

$$
\left(\tau \partial_{t}+r\right) C(t)=\tilde{\mu} \int_{-\infty}^{t} d \bar{t} e^{-\alpha(t-\bar{t})} C(t-\bar{t}) C(\bar{t})+\mu \int_{0}^{\infty} d \bar{t} e^{-\alpha(t+\bar{t})} C(t+\bar{t}) G(\bar{t})
$$




$$
\left(\tau \partial_{t}+r\right) G(t)=\tilde{\mu} \int_{0}^{t} d \bar{t} e^{-\alpha(t-\bar{t})} C(t-\bar{t}) G(\bar{t})
$$

Here the difference of the times $t-t^{\prime}$ is denoted by the same letter $t$. We also take $\tau=1$ as fixation of the units.

Further, we shall investigate the adiabatic limit, where the bonds are frozen with respect of the dynamics of spins [6, 13]. Indeed, in a qualitative manner we feel that any spin-glass order is possible only if bonds are frozen. This intuitively obvious fact has been rederived rigorously recently for spin-glass systems when the detailed balance condition holds [8, 13]. Thus the limit

$$
\tau_{J} \gg t-t^{\prime}
$$

is assumed: The bonds are frozen when the spins are observed. Actually, this assumption is the standard one for investigating the dynamics of usual spin-glass models when the bonds are frozen a priori. The different factor of our approach is the noninfinite temperature $T_{J}$, which is a physical mechanism for introducing a correlation between bonds.

Now if $t-t^{\prime}$ is large enough, but stills much smaller than $\tau_{J}$ and all these times are much smaller than modulo of the initial time which $\rightarrow-\infty$ just after $N \rightarrow \infty$, we expect that the correlation function tends to the Edwards-Anderson order parameter

$$
C\left(t-t^{\prime} \mapsto \infty\right)=q
$$

By the adiabatic condition (6) in the last integral of Eq. (四) we can take $e^{-\alpha \bar{t}} \sim 1$ by considering the relevant domain of the integration. In the first integral we have by interchanging variable

$$
\tilde{\mu} \int_{0}^{\infty} d \bar{t} e^{-\alpha \bar{t}} C^{p-1}(\bar{t}) C(t-\bar{t}) \mapsto \frac{\beta q^{2}}{v}
$$

Similar analysis shows that the right-hand term in Eq. (5) can be neglected, and we have the simple solution

$$
G(t)=e^{-r t}
$$

There are three relevant parameters in our model: $v, \beta, \beta_{J}$. In this model we consider the phase diagram at the following conditions: $v=T_{J}, n=T / T_{J}$ is fixed, and only $\beta=1 / T$ 
is varying. Thus by

$$
n \mapsto 0
$$

we go to the model was considered in [9]. 2 This fact can be derived directly from (2): By limits (6, 9) the bonds can be considered as uncorrelated, quench, asymmetric gaussian variables.

After this we have the following equation for $C(t)$ :

$$
\left(\partial_{t}+r\right) C(t)=n \beta^{2} q^{2}+\beta^{2} \int_{0}^{\infty} d \bar{t} C(t+\bar{t}) e^{-r \bar{t}}
$$

This equation holds for $t \geq 0$.

Equation (10) can be solved by the Laplace transformation. As the general solution we have

$$
C(t)=\frac{n \beta^{2} q^{2} r}{r^{2}-\beta^{2}}+k_{+} e^{t \sqrt{r^{2}-\beta^{2}}}+k_{-} e^{-t \sqrt{r^{2}-\beta^{2}}} .
$$

The constants $k_{+}, k_{-}, r$ should be fixed by the following standard conditions: $C(0)=1$ (the spherical constraint), $\left.\partial_{t} C(t)\right|_{t=0}=-1, k_{+}=0$ (the condition for monotonic decay of $C(t)$ ). After some calculations we have for $r$ and Edwards-Anderson parameter $q$

$$
\begin{gathered}
q=\frac{n \beta^{2} q^{2} r}{r^{2}-\beta^{2}} \\
r=\sqrt{\beta^{2}+\frac{1}{(1-q)^{2}}} .
\end{gathered}
$$

Finally we have the following equation for $q$ :

$$
\frac{q}{1-q}=n \beta^{2} q^{2} \sqrt{\beta^{2}(1-q)^{2}+1}
$$

Besides paramagnetic solution $q=0$ there is also the first-order phase transition into a spin-glass phase (it is obvious that second-order transition is impossible in this case). The transition point is defined as the first temperature when (14) predicts a non-zero

\footnotetext{
${ }^{2}$ Furthermore, if the bonds in (1, 2) are symmetric a priori then by (6, 9) we go to the usual spherical mean-field model [13]. Here $n$ plays the role of "replica number" which in other approaches is introduced by the replica trick.
} 
(non-paramagnet) solution. The temperature and the jumping of $q$ at this transition point can be obtained by the following equations:

$$
\begin{aligned}
& q_{c}=\frac{\psi+1}{3 \psi+2}, \\
& T_{c}=\frac{1-q_{c}}{\sqrt{\psi}},
\end{aligned}
$$

where $\psi$ is the positive solution of the equation

$$
2 \psi+1=n \psi(1+\psi)^{3 / 2} .
$$

When $n \mapsto 0$ (in this limit we go to the particular case that was studied in Ref. [9]) we have $T_{c} \mapsto 0, q_{c} \mapsto 1 / 3$.

Further, we should discuss the stability properties of the spin-glass solution. If detailed balance holds, then static properties of a system is described by the Gibbs distribution therefore the stability of solutions can be investigated by analyzing minimums of free energy [3. But for this concrete problem free energy is not necessary: Unstability of a solution is reflected by unstable behavior of purely dynamical quantities such as positive derivation of correlation function, or negative susceptibility. For example, the famous (AT) line in usual spin-glass models can be recovered by this purely dynamical consideration [12, 17]. This property does not connect with any specific character of spin-glass systems but is supported by general theorems about the stability of stochastic dynamical systems (generally speaking without detailed balance) [1, 2, 7]. Because in our case there are no such anomalies both for paramagnet and spin-glass solutions we conclude that these phases are stable (by chosen initial conditions). The stability of paramagnet for any temperature is more or less typical for first-order phase transitions [11, 14, 13].

Now about an other important problem: If we have two different stable phase, then one should be only metastable and another one true stable. In usual models this question

\footnotetext{
${ }^{3} \mathrm{~A}$ remark should be given here: Because the mean-field dynamical equations are obtained by $N \rightarrow \infty$ before the initial time $\rightarrow-\infty$, some states that arise in dynamics cannot be reflected in the purely static investigation by means of free energy. Thus, phase transitions that are predicted by statics and dynamics can be different 12, 14,
} 
is investigated by free energy: For the true stable phase the free energy should be minimal. In principle, a generalized free energy can be introduced also in models without detailed balance; the corresponding quantity is based on the so called Kullbak entropy or relative entropy [1, 2]. For systems with non-gibbsian stationary distribution this function plays nearly the same role as usual free energy but, generally speaking, has a strong dependence from initial conditions - in the usual case the detailed balance condition removes this dependence. Unfortunately, we have not succeeded in calculation of this function for our model. So really we found only that the spin-glass phase occur at least as a metastable state. On other hand all stable spin-glass solutions - known by other models (see, for example, [12, 13, 18]) - are true stable with respect to paramagnet at least if temperature is low enough (of course it is not an argument for true stability in our case but rather a hint). One of the scenarios for phase transitions realized in such systems is as follows [13, 15]: At some temperature the nontrivial phase (spin-glass in our case) occurs at the first time, but true thermodynamical transition occurs at some lower temperature where free-energies of the different solutions are the same. May be the scenario for our phase transition is the same.

In the low-temperature limit we have for our solution

$$
1-q \sim \frac{T^{2}}{n}
$$

We get this equation by the assumption that $1-q$ is small. It should be noted that the characteristic time for the correlation function relaxation is $t_{r e l}=1-q$. Thus, critical slowing down occur only near $T=0$.

Now about the origin of this spin-glass phase. As we mentioned above behavior of a system without detailed balance can be connected with behavior of the phase space of the same system when detailed balance holds (i.e., the bonds are symmetric in our case). If we assume that such arguments can be used, then at this qualitative level the situation is clear: as was shown in [13] a finite $n$ breaks marginality of the spin-glass solution in the two-spin spherical model. 'Replicon' (or dangerous) eigenvalue is $\sim n$ (except exactly the critical point). Thus, at least, some spin-glass states are totally stable, and can 'struggle' 
against the nonhamiltonian influence.

We consider the spherical spin-glass model with asymmetric partially annealed bonds and show that this last property can induce a transition to the spin-glass phase. Several very important problems in this field should be considered in future: The problems of

proving true stability for the new phase, considering partially asymmetric bonds, and of course investigating of more realistic models.

\section{Acknowledgments.}

Authors thank unknown referee for physically very well-motivated remarks. A.E. Allahverdyan also thank E. Mamasakhlisov for helpful discussions.

\section{References}

[1] R.L. Stratanovich, Nonequilibrium, Nonlinear Thermodynamics, Moscow, Nauka, 1985.

[2] F. Shlogl, Phys. Rep.,62, 268, (1980).

[3] V.G. Gorshkov, Physical and Biological Bases of Life stability., Springer-Verlag, 1994.

[4] G. Parisi, J. Phys. (A),19, L675, (1986).

[5] For a comprehensive review and further references about nonequilibrium steady states, see: B. Schmittmann and R.K.P. Zia, in Phase Transitions and Critical Phenomena, eds. C. Domb and J.L. Lebowitz (Academic Press, New York, 1996).

[6] Gardiner, Handbook of Stochastic Methods, Springer-Verlag, 1982.

[7] P. Hanggri, H. Thomas, Phys. Rep.,88, 209, (1982).

[8] H. Horner, Z. Phys. B, 57, 29, (1984); ibid, 57, 39, (1984).

[9] A. Crisanti, H. Sompolinsky, Phys.Rev.(A), 36, 4922, (1987). 
[10] L. F. Cugliandolo, J. Kurchan, P. Le Doussal, and L. Peliti, Phys. Rev. Lett . 78, 350 (1997).

[11] A. Crisanti and H.-J. Sommers, Z. Phys. B87, 341 (1992).

[12] A. Crisanti, H. Horner and H.-J. Sommers, Z. Phys. B92, 257 (1993).

[13] A.E. Allahverdyan, T.M. Nieuwenhuizen, D.B. Saakian, unpublished. J.Phys. (A), 26, 3681, (1993).

[14] L.F. Cugliandolo and J. Kurchan Phys.Rev.Lett., 71, 173, (1993), Phil.Mag.B71, $50,(1995)$.

[15] E. Nagaev, Magnetics with Complicate Exchange Interaction, Moscow, Nauka, 1988. (1995).

[16] H.K. Janssen, Z. Phys. B 26, 187 (1977); C. De Dominicis and L. Peliti, Phys. Rev. Lett. 38, 505 (1977); Phys. Rev. B 18, 353 (1978)

[17] H. Sompolinsky, and A. Zippelius, Phys. Rev. B, 25, 6860, (1982).

[18] D.J. Gross, I. Kanter, and H. Sompolinsky, Phys. Rev. Lett. 55, 304, (1985); T.R. Kirkpatrick and D. Thirumalai, Phys.Rev. B, 36, 5388, (1987). 